EPJ manuscript No.

(will be inserted by the editor)

\title{
Lattice bosons in quartic confinement
}

\author{
R. Ramakumar ${ }^{1 \mathrm{a}}$ and A. N. Das ${ }^{2}$ \\ 1 Department of Physics and Astrophysics, University of Delhi, Delhi-110007, India \\ 2 Theoretical Condensed Matter Physics Division, Saha Institute of Nuclear Physics, 1/AF Bidhannagar, Kolkata-700064, India
}

12 January 2008

\begin{abstract}
We present a theoretical study of bose condensation of non-interacting bosons in finite lattices in quartic potentials in one, two, and three dimensions. We investigate dimensionality effects and quartic potential effects on single boson density of energy states, condensation temperature, condensate fraction, and specific heat. The results obtained are compared with corresponding results for lattice bosons in harmonic traps.
\end{abstract}

PACS. 0 3.75.Lm, 03.75.Nt, 03.75.Hh

\section{Introduction}

Over last few years, bosons and fermions in optical lattices

have emerged as important controllable systems for inves-

tigations into several properties of quantum many-particle

systems [1. These are clean systems in which experimen-

talists have achieved great control over a wide range of particle numbers, particle hopping, and strength and sign of inter-particle interactions. Experimental groups have conducted extensive studies $2,3,4,5,6,7,8,8$ of several properties of many-boson systems in one, two, and three dimensional optical lattices. The many-boson system in these experiments is under the combined influence

\footnotetext{
${ }^{\mathrm{a}}$ e-mail: rkumar@physics.du.ac.in
}

of the periodic lattice potential and an overall confining harmonic potential. Many theoretical studies $9,10,11,12$ 13, 14, 15, 16, 17, 18, 19, 20 of such lattice bosons in harmonic confinement have appeared in recent years. Unlike the case of lattice bosons in harmonic confinement, the effect of anharmonic potentials on the properties of lattice bosons have begun to be explored only recently 21. In that work Gygi and collaborators studied zero temperature properties of strongly interacting lattice bosons in a quartic trap. It has been suggested 21] that it is experimentally possible to create an optical lattice in a quartic trap employing a combination of red-detuned and bluedetuned Gaussian laser beams. Finite temperature prop- 
erties of lattice bosons in a quartic trap is of considerable interest in this context.

In this paper, we present a theoretical study of lattice bosons in a quartic potential in one, two, and three dimensions (1d, 2d, and 3d). We consider non-interacting bosons in a periodic lattice in $1 \mathrm{~d}$ in a $1 \mathrm{~d}$ quartic potential, a square lattice in a $2 \mathrm{~d}$ quartic potential, and a cubic lattice in a $3 \mathrm{~d}$ quartic potential. We study the effects of the potential on one-boson density of energy states (DOS) and the temperature dependence of ground state occupancy and specific-heat. We compare the results obtained for lattice bosons in quartic traps to the corresponding results for lattice bosons in harmonic traps 20. This work is presented in the Sections 2 and 3, and conclusions are given in Section 4. In the work presented in the following sections, we have not included the effect of boson-boson interaction $(U)$. Our results would approximately also hold in the weak interaction regime $(U<<t$, where $t$ is the boson hopping energy) where the interaction induced depletion effects are not significant 22. A weakly interacting regime may be achieved by adjusting the lattice potential depth to a low value as has already been done for lattice bosons in quadratic traps 2,4].

\section{Model and method}

In this Section, we give a brief presentation of the model of the system and the method followed in the calculations of various properties presented in later sections. The Hamil- tonian of the many-boson system we consider is

$$
H=-t \sum_{<i j>}\left(c_{i}^{\dagger} c_{j}+c_{j}^{\dagger} c_{i}\right)+\sum_{i} V(i) n_{i}-\mu \sum_{i} n_{i}
$$

where $t$ is the kinetic energy gain when a boson hop from site $i$ to its nearest neighbor site $j$ in the optical lattice, $c_{i}^{\dagger}$ is the boson creation operator, $V(i)$ is the quartic potential at site $i, n_{i}=c_{i}^{\dagger} c_{i}$ the boson number operator, and $\mu$ the chemical potential. The forms of the quartic potentials used are: $V(i)=Q x_{i}^{4}$ in $1 \mathrm{~d}, V(i)=Q\left(x_{i}^{4}+y_{i}^{4}\right)$ in $2 \mathrm{~d}$, and $V(i)=Q\left(x_{i}^{4}+y_{i}^{4}+z_{i}^{4}\right)$ in $3 \mathrm{~d}$. We first obtain the matrix representation of the system Hamiltonian in a site basis. We numerically diagonalize it to obtain energy levels of a lattice boson. We have used open boundary conditions. We have chosen lattice sizes large enough so that finite size effects are absent in the results presented in the next section. The lattice sizes were fixed by finding the lattice size beyond which results remain unchanged with further increase of lattice size. This depends on the magnitude of the quartic potential strength since it decides the spread of the boson distribution in the lattice for a given value of $t$ and temperature. The energy levels $\left(E_{i}\right)$ obtained for a boson in these large lattices are used in calculations of the DOS, ground state occupancy, and the specific heat. The chemical potential and boson populations in the various energy levels are calculated using the boson number equation: $N=\sum_{i=0}^{m} N\left(E_{i}\right)$, where $E_{0}$ and $E_{m}$ are the lowest and the highest single boson energy levels and $N\left(E_{i}\right)=1 /\left[\exp \left[\beta\left(E_{i}-\mu\right)\right]-1\right]$ in which $\beta=1 / k_{B} T$ with $k_{B}$ the Boltzmann constant and $T$ the temperature. The specific heat is calculated from the temperature derivative 
of total energy $\left(E_{t o t}=\sum_{i=0}^{m} N\left(E_{i}\right) E_{i}\right)$. All energies are measured in units of $t$.

\section{Results and Discussion}

\subsection{One-boson density of energy states}

In this section, in Figs. 1-4, we present our results on the one-boson density of energy states (DOS) for a lattice bosons in a quartic trap and compare with the corresponding DOS for a lattice boson in a harmonic trap for which: $V(i)=K\left(x_{i}^{2}+y_{i}^{2}+z_{i}^{2}\right)$. In Fig. 1, we have exhibited the DOS for bosons in optical lattices with harmonic and quartic confinement potentials for several values of $q=Q a^{4}$ and $k=K a^{2}$, where $a$ is lattice constant. We find that the confining potential has a significant effect on the DOS in both cases. In the case of quartic confining potential (Fig. 1(a)), the divergence in DOS at the band edges is found to be suppressed and eventually destroyed with increasing strength of the potential. Further, the quartic potential spreads the DOS over a wide energy scale. It is also to be noted that for small potential strengths shown in the figure, the low energy part of the DOS continues to show similarity to the case of the DOS of lattice bosons without confinement. In comparison, in the case of harmonic potential (Fig. 1(b)), divergence of the DOS at the lower edge of the band is destroyed while the one at higher edge is significantly suppressed even for a weak harmonic potential, and with increasing strength the DOS is flattened to a wide energy scale 23. In Fig. 2, we have shown our results on the DOS of a boson in a $2 \mathrm{~d}$ square lattice in a $2 \mathrm{~d}$ quartic potential (Fig. 2(a)) and the corresponding results for the harmonic case(Fig. 2(b)). In the quartic potential case, we find that the vanHove singularity is strongly suppressed by the confinement potential. In comparison, the Van Hove singularity is destroyed by the harmonic potential[23]. In both cases the confining potentials spread the DOS compared to the pure lattice case. Fig. 3 shows the DOS of a boson in a cubic lattice in a quartic potential. Increasing the quartic potential strength clearly has a strong effect on the DOS. Similar results are obtained for the harmonic potential case as well, as shown in Fig. 4. The dotted lines in Fig. 4 are the single particle DOS for an infinite dimensional hypercubic lattice whose DOS is [24]: $\rho(E)=\exp \left[-E^{2} /\left(2 t^{2}\right)\right] / \sqrt{2 \pi t^{2}}$. On comparing the changes brought about by the harmonic potential in $1 \mathrm{~d}, 2 \mathrm{~d}$, and $3 \mathrm{~d}$, we notice an approximate dimensionality crossover in the DOS for small $k$. For a $1 \mathrm{~d}$ lattice with harmonic potential, the DOS has a finite value at the lower band edge and a weak singularity well inside the band, which are characteristics of the DOS of a $2 \mathrm{~d}$ lattice in the absence of confining potential. For a $2 \mathrm{~d}$ lattice with harmonic potential, the DOS almost vanishes at the lower band edge and has a flat region in the middle part of the band, which are characteristics of the DOS of a $3 \mathrm{~d}$ lattice in the absence of any confining potential. Finally, the DOS of a 3d lattice with harmonic potential is found to be close to that of an infinite dimensional hypercubic lattice. Hence in DOS, the dimensionality crossovers seen are: $1 d(k \neq 0) \rightarrow 2 d(k=0), 2 d(k \neq 0) \rightarrow 3 d(k=0)$, and $3 d(k \neq 0) \rightarrow \infty d(k=0)$. In comparison, such dimension- 
ality crossovers are not found in the case of lattice bosons in quartic traps.

\subsection{Ground state occupancy and specific heat}

In this section we present our results on condensate fraction, condensation temperature, and specific heat of lattice bosons in quartic potentials. A comparison of the temperature dependence of the fractional ground state occupancy for 1d lattice bosons in a 1d harmonic trap and in a 1d quadratic trap is shown in Fig. 5 where we have plotted the variation of $N_{0} / N$ with $T / T_{0}$. Here $N_{0}$ is the boson population in the lowest energy level and $T_{0}$ is determined by setting $N_{0}=0$ and $\mu=E_{0}$ in the number equation (i.e., by solving $\left.\left.N=\sum_{i=1}^{i_{m}} 1 /\left[\exp \left(E_{i}-E_{0}\right) / k_{B} T_{0}\right)-1\right]\right)$. We note that the dependence of fractional ground state occupancy on scaled temperature $\left(T / T_{0}\right)$ is nearly independent of the strengths of the potentials when lattices are large enough that finite size effects are absent. The lattice sizes and the strengths of potentials we have used satisfy this condition. The dependence of $T_{0}$ on the strength of the quartic potential presented in Fig. 6 shows a fast increase for small values of $q$ and a monotonic increase for larger $q$. The boson number dependence of $T_{0}$ is found to be linear (not shown) similar to lattice bosons in a harmonic potential 20]. In Fig. 7, we have shown the dependence of the specific heat on scaled temperature. Increasing the the strength of quartic potential suppress $C_{v}$ except in the low temperature regime. In the low temperature range, the $C_{v}$ is nearly independent of the potential strength. This can be qualitatively understood if we regard the DOS in Fig. 1(a). At low temperatures the bosons are in low energy states. The DOS plots implies that change in the shape of the band bottom is not significant for small $q$. Hence for small $q$, the $C_{v}$ curves for different $q$ values are very close to each other in the low temperature range.

In Figs. 8-10, we have presented our results for bosons in $2 \mathrm{~d}$ square lattices in a $2 \mathrm{~d}$ quartic potential. Compared to the $1 \mathrm{~d}$ case, the condensate fraction is found to increase faster with decreasing temperature in the temperature range below $T_{0}$. The temperature variation of $N_{0} / N$ is seen to be close to the case of pure lattice bosons compared to lattice bosons in a harmonic trap. This feature is also found in the $3 \mathrm{~d}$ case discussed later. The results of the quartic confinement case is closer to the pure lattice case since, in the central region of the lattice, the quartic potential is shallower compared to the harmonic confining potential. In the low energy range, the DOS of a lattice with quartic potential is closer to that of a pure lattice compared to the DOS of a lattice with harmonic confining potential. Increase in dimensionality leads to smaller number of bosons in the ground state for $T \geq T_{0}$. Fig. 9 shows the dependence of condensation temperature on the strength of the quartic potential. While the $k$ dependence is similar to that found in $1 d$, the magnitude of $T_{0}$ is much lower in $2 \mathrm{~d}$ compared to that in $1 \mathrm{~d}$ for the same value of $q$. Fig. 10 shows the dependence of specific heat on temperature for various strengths of the quartic trap potential. Unlike in $1 \mathrm{~d}$, the $C_{v}$ is found to have a peak near the condensation temperature. In the intermediate to high temperature range, the specific heat is found 
to be suppressed with increasing potential strength. Low temperature $C_{v}$ shows a slight enhancement with increasing $q$. When compared with the low temperature $C_{v}$ of lattice bosons in harmonic traps which has a $\left(T / T_{0}\right)^{2}$ dependence, we find that this low temperature part for the quartic trap has approximately a $\left(T / T_{0}\right)^{1.7}$ dependence as shown in Fig. 11. This can be understood when we consider the DOS of a lattice boson in quartic and harmonic traps. We first recall that the temperature dependence of the specific heat of bosons goes as $\left(T / T_{0}\right)^{\alpha}$ for a $E^{(\alpha-1)}$ dependence of the DOS 25]. Now, we find that the low energy part of the DOS of lattice bosons in a quartic trap can be fitted with a value of $\alpha \approx 0.8-0.9$, as a consequence of which the specific heat exponent is less than 2. For $2 \mathrm{~d}$ bosons in a $2 \mathrm{~d}$ harmonic trap, low energy part of DOS has a linear $E$ dependence leading to a $\left(T / T_{0}\right)^{2}$ dependence of $C_{v}$.

Now we discuss our results on 3 d lattice bosons in a $3 \mathrm{~d}$ quartic trap. The temperature dependence of the condensate fraction, shown in Fig. 12, shows that increased dimensionality makes the condensation sharper. The Bose condensation is favored by small values of the DOS at the bottom of the energy band. With increasing dimensionality, the DOS decreases at and near the bottom of the band and this leads to the sharpness of the condensation. Similar to $1 \mathrm{~d}$ and $2 \mathrm{~d}$ results, the growth of the condensate fraction with temperature is found to lie intermediate between the lattice bosons in a harmonic trap and pure lattice bosons. Clearly, if one is looking for phases of bosons in optical lattices with minimum intervention from the overall confining potential, it would be better to use a quartic confinement rather than a harmonic one. Fig. 13 contains our results on quartic potential strength dependence on the condensation temperature. These results are qualitatively similar to $1 \mathrm{~d}$ and $2 \mathrm{~d}$, and quantitatively similar to $2 \mathrm{~d}$ than $1 \mathrm{~d}$. The temperature dependence of $C_{v}$ is shown in Fig. 14 for various strengths of the quartic potential. Increased dimensionality makes the peak at the condensation temperature sharper. Similar to $1 \mathrm{~d}$ and $2 \mathrm{~d}$, increasing the strength of the potential leads to suppression of $C_{v}$ except in the low temperature region. The low temperature specific heat has a $\left(T / T_{0}\right)^{2.65}$ dependence as shown in Fig. 11. For lattice bosons in a harmonic trap, the low temperature $C_{v}$ has a $\left(T / T_{0}\right)^{3}$ dependence. This difference originates from the difference in the DOS for these two cases which, in the low temperature range, shows a $E^{(\alpha-1)}$ dependence with $\alpha=3$ for lattice bosons in a harmonic trap and $\alpha \approx 2.5-2.6$ for the quartic trap.

\section{Conclusions}

In this paper, we presented results of our calculations of single boson density of energy states, condensate fraction, condensation temperature, and specific heat of bosons in some one, two, and three dimensional periodic lattices in a quartic potential. Wherever possible, the results obtained are compared with the corresponding results for lattice bosons in harmonic traps. In one dimension, we find that the DOS of a lattice boson in a quartic potential continues to retain its pure lattice form for small potential strengths unlike the case of harmonic trap. In two dimensions, the 
Van Hove singularity is suppressed but not eliminated for small quartic potential strengths in contrast to the effect of a harmonic potential. In three dimensions, the quartic and harmonic potential are found to destroy flat regions of the DOS of a pure lattice. In addition, for lattice bosons in a harmonic potential, one finds a dimensionality crossover in the DOS, which is not found in the case of a quartic potential. The temperature variation of condensate fraction, condensation temperature, and specific heat is found to be intermediate between those of lattice bosons in a harmonic potential and pure lattice bosons.

\section{Acknowledgments}

RRK thanks Professor Bikash Sinha, Director, SINP and Professor Bikas Chakrabarti, Head, TCMP Division, SINP for hospitality at SINP. RRK also thanks Professor Helmut Katzgraber, ETH, Zurich for drawing his attention to bosons in quartic traps. We thank Dr. S. Sil, Visva Bharati, Santiniketan for help in computation.

\section{References}

1. I. Bloch, J. Dalibard, W. Zwerger, arXiv:0704.3011v1 [condmat.other].

2. M. Greiner, O. Mandel, T. Esslinger, T. W. Hänsch, I. Bloch, Nature 415, 39 (2002).

3. T. Stöferle, H. Moritz, C. Schori, M. Köhl, T. Esslinger, Phys. Rev. Lett. 92, 130403 (2004).

4. C. Schori, T. Stöferle, M. Henning, M. Köhl, T. Esslinger, Phys. Rev. Lett. 93, 240402 (2004).
5. B. Paredes, A. Widera, V. Murg, O. Mandel, S. Fölling, I. Cirac, G. V. Shlyapnikov, T. W. Hänsch, I. Bloch, Nature 429, 277 (2004).

6. M. Köhl, H. Moritz, T. Stöferle, C. Schori, T. Esslinger, J. Low Temp. Phys. 138, 635 (2005).

7. G. K. Campbell, J. Mun, M. Boyd, P. Medley, A. E. Leanhardt, L. G. Maracassa, D. E. Pritchard, W. Ketterle, Science 313, 649 (2006).

8. S. Fölling, A. Widera, T. Müller, F. Gerbier, I. Bloch, Phys. Rev. Lett. 97, 060403 (2006).

9. D. Jaksch, C. Bruder, J. I. Cirac, C. W. Gardiner, P. Zoller, Phys. Rev. Lett. 81, 3108 (1998).

10. W. Zwerger, J. Opt. B: Quantum Semiclass. Opt. 5, S9 (2003).

11. A. M. Rey, K. Burnett, R. Roth, M. Edwards, C. J. Williams, C. W. Clark, J. Phys. B: At. Mol. Opt. Phys. 36, 825 (2003)

12. G. Pupillo, E. Tiesinga, C. J. Williams, Phys.Rev. A 68, 063604 (2003).

13. S. Wessel, F. Alet, M. Troyer, G. G. Batrouni, Phys. Rev. A 70, 053615 (2004).

14. L. Pollet, S. Rombouts, K. Heyde, J. Dukelsky, Phys. Rev. A 69, 043601 (2004).

15. S. M. Giampaolo, F. Illuminati, G. Mazzarella, S. De Siena, Phys. Rev. A 70, 061601 (2004).

16. B. DeMarco, C. Lannert, S. Vishveshwara, T.-C. Wei, Phys. Rev. A 71, 063601 (2005).

17. G. Pupillo, A. M. Rey, G. G. Batrouni, Phys. Rev. A 74, 013601 (2006).

18. B. G. Wild, P. B. Blakie, D. A. W. Hutchinson, Phys. Rev. A 73, 023604 (2006). 
19. V. Murg, F. Verstraete, J. I. Cirac, Phys. Rev. A 75, 033605 (2007).

20. R. Ramakumar, A. N. Das, S. Sil, Eur. Phys. J. D 42, 309 (2007)

21. O. Gygi, H. G. Katzgraber, M. Troyer, S. Wessel, G. G. Batrouni, Phys. Rev. A 73, 063606 (2006).

22. R. Ramakumar and A. N. Das, Phys. Rev. B 72, 094301 (2005).

23. C. Hooley and J. Quintanilla, Phys. Rev. Lett. 93, 080404 (2004).

24. W. Metzner and D. Vollhardt, Phys. Rev. Lett. 62, 324 (1989).

25. C. J. Pethick and H. Smith, Bose-Einstein condensation in dilute gases (Cambridge University Press, Cambridge, England, 2002).
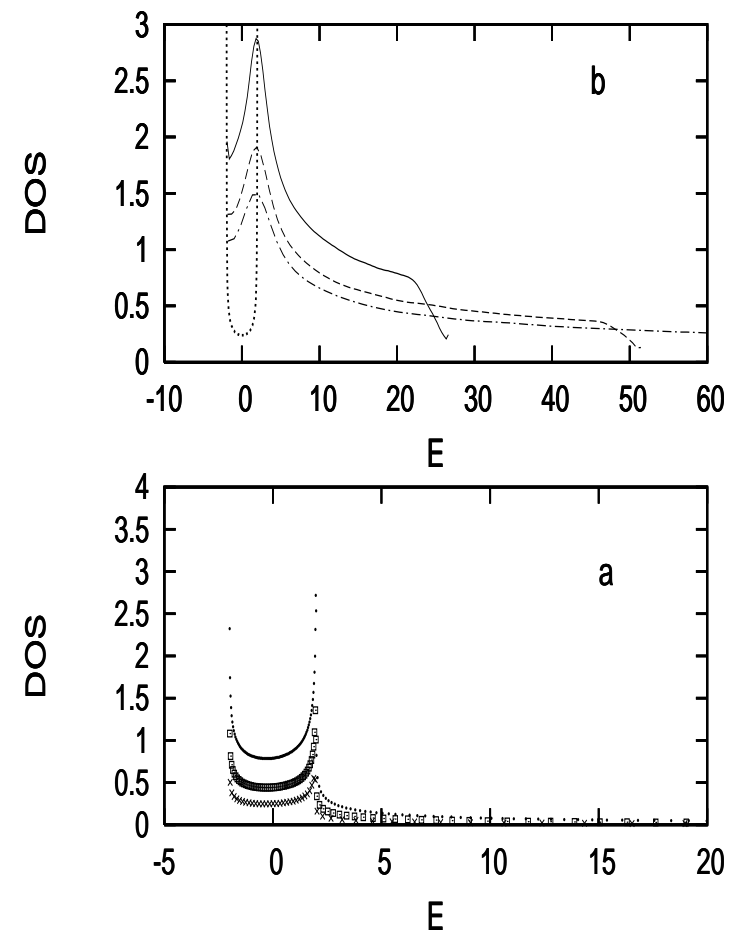

Fig. 1. Density Of States (DOS) of a boson in a onedimensional periodic lattice of size 1000 in quartic (a) and harmonic (b) potentials. In bottom panel (a): $q=0.25 \times 10^{-6}$ (dots), $0.25 \times 10^{-5}$ (squares), and $0.25 \times 10^{-4}$ (crosses). In top panel (b): $k=0$ (dots), 0.0001 (solid), 0.0002 (dashes), and 0.0003 (dash-dot). All energies are measured in units of $t$. 

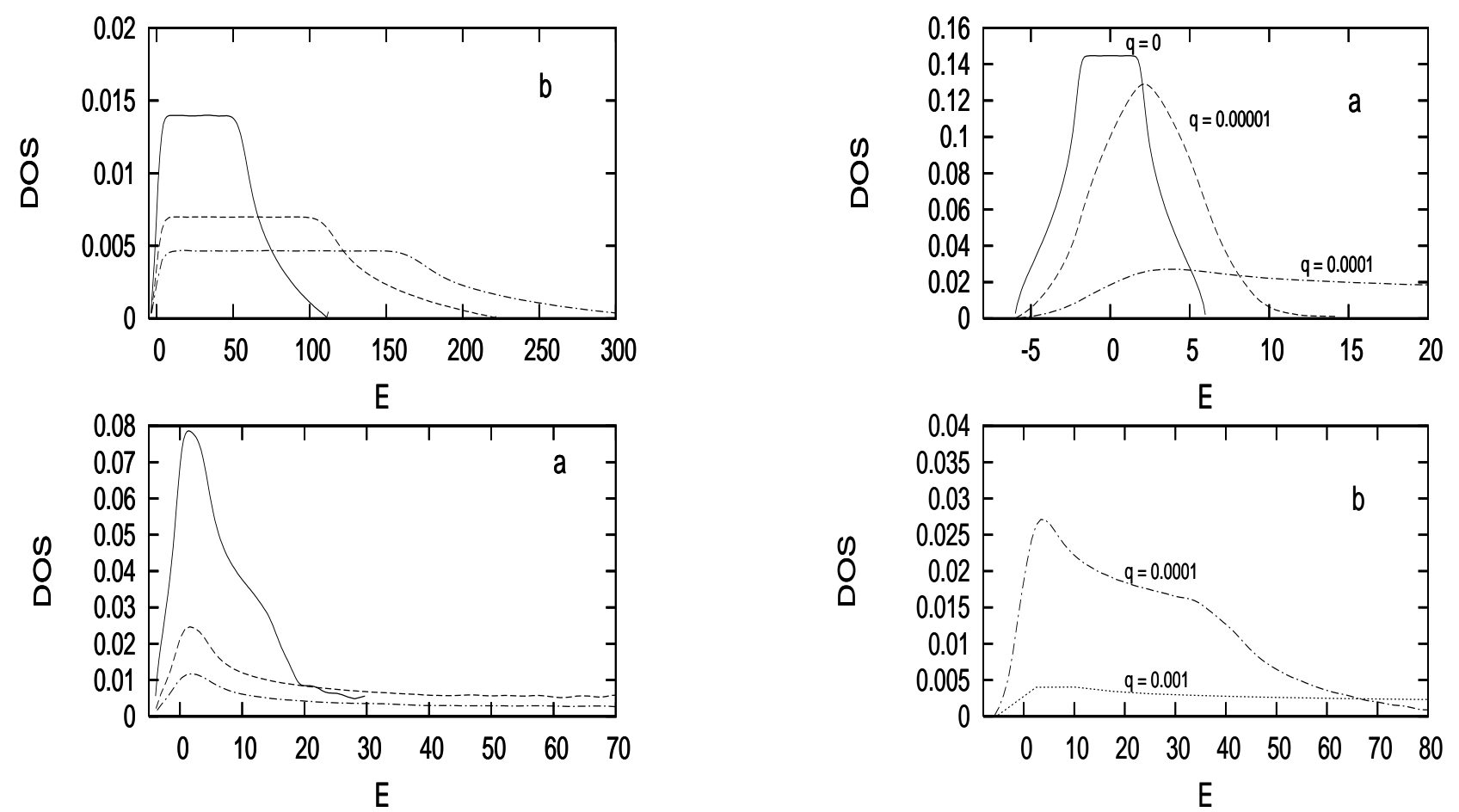

Fig. 2. The DOS of a boson in a two-dimensional square

Fig. 3. The effect of quartic potential strength on the DOS lattice of size $150 \times 150$ in a quartic (a) and harmonic (b) of a boson in a three-dimensional cubic lattice of size $50 \times 50$ potentials. In the bottom panel (a): $q=0.25 \times 10^{-5}$ (solid), $\times 50$.

$0.25 \times 10^{-4}$ (dashes), and $0.10 \times 10^{-3}$ (dash-dot). In top panel (b): $k=0.01$ (solid line), 0.02 (dashed line), and 0.03 (dashdot). 


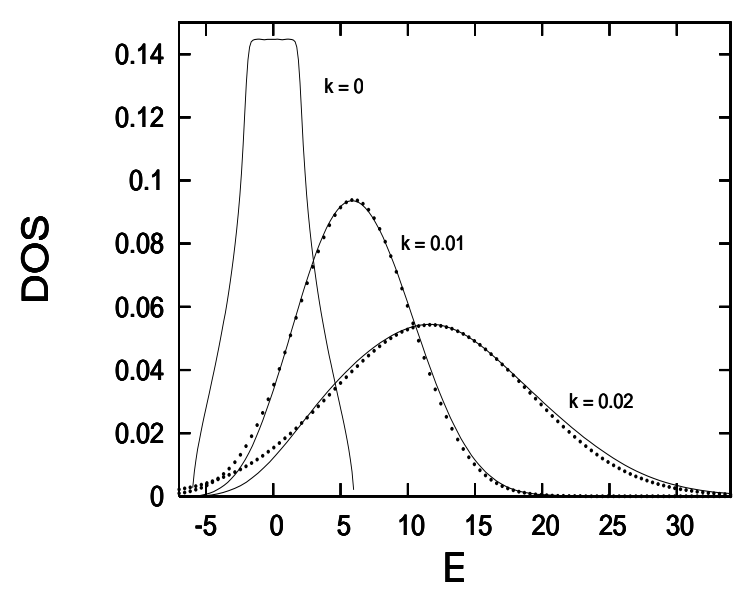

Fig. 4. The effect of the strength of harmonic potential on DOS of a boson in a three-dimensional cubic lattice of size 70 $\times 70 \times 70$ for the values of $\mathrm{k}$ shown on the curves. The dotted lines are DOS's for an infinite dimensional lattice in the absence of any confining potential, as discussed in the text.

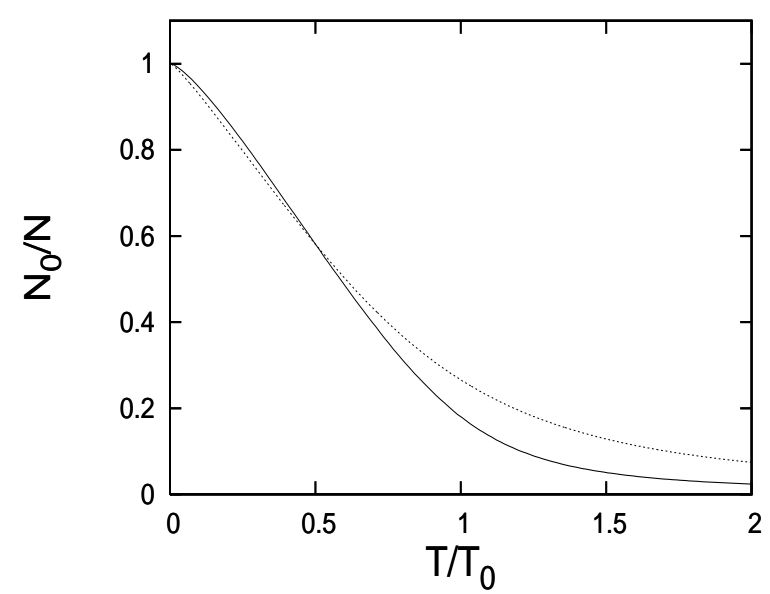

Fig. 5. Temperature dependence of condensate fraction for bosons in $1 \mathrm{~d}$ optical lattices with confining potentials: lattice bosons in a quartic trap of strength $q=0.25 \times 10^{-6}$ (dots) and lattice bosons in a harmonic trap of strength $\mathrm{k}=0.001$ (solid). The lattice size used is 1000 and the number of bosons is 600 . The values of $k_{B} T_{0}$ for these cases are 3.23 , and 6.773 , respectively.

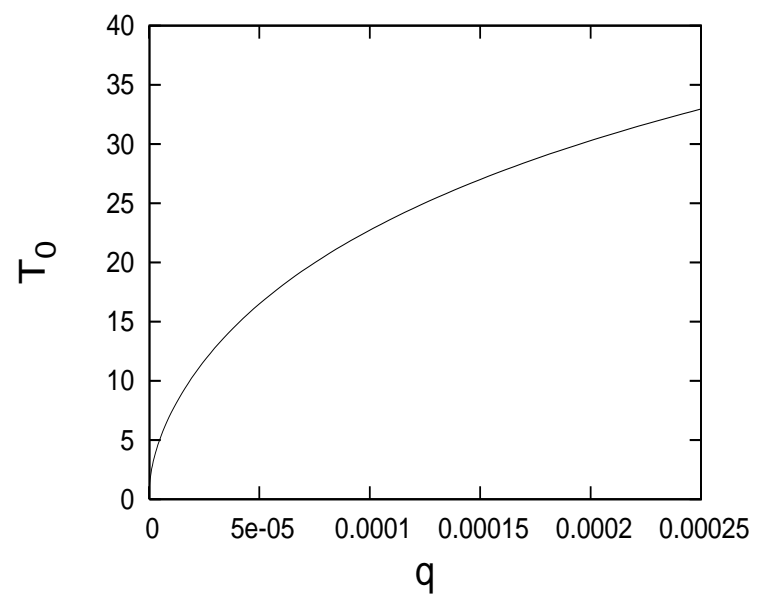

Fig. 6. Quartic potential strength dependence of condensation temperature for 600 bosons in a 1d periodic lattice of size 1000 . The lowest value of $\mathrm{q}$ in this figure is $0.25 \times 10^{-6}$.

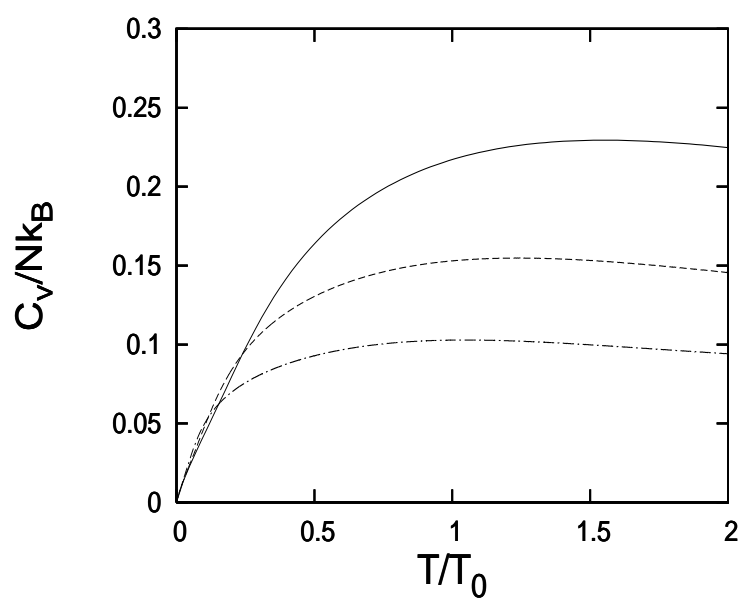

Fig. 7. Temperature dependence of the specific heat of 600 bosons in a 1d lattice of size 1000 in a quartic potential of stregnths $q=0.25 \times 10^{-6}\left(\right.$ solid line), $0.25 \times 10^{-5}(\operatorname{dots})$, and $0.25 \times 10^{-4}$ (dash-dot). The values of $k_{B} T_{0}$ for these cases are $3.23,6.91$, and 14.98 , respectively. 


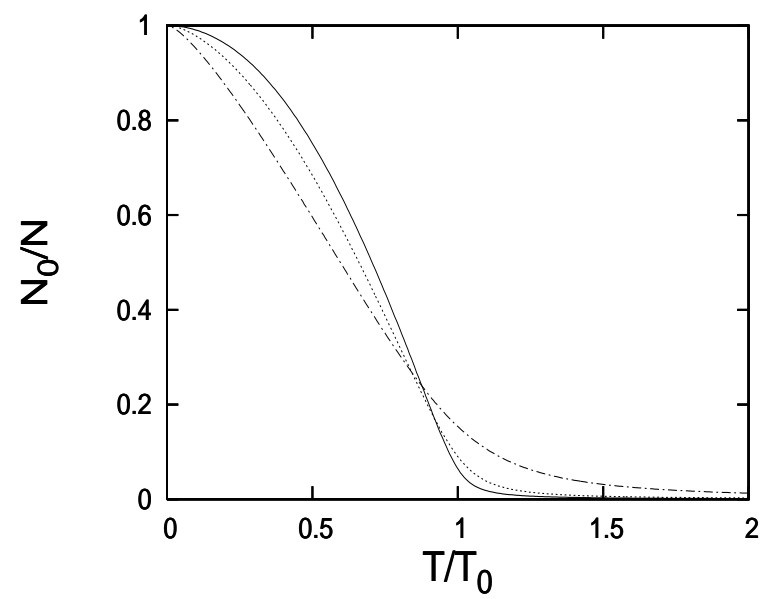

Fig. 8. Comparison of the growth of condensate fraction of lattice bosons in $2 \mathrm{~d}$ : bosons in a $2 \mathrm{~d}$ square lattice of size 100 $\times 100$ (dash-dot), lattice bosons in a quartic trap of strength $q=0.25 \times 10^{-5}$ (dotted), and lattice bosons in a harmonic trap of strength $\mathrm{k}=0.01$ (solid). The lattice sizes used are $150 \times 150$ (harmonic trap case) and $100 \times 100$ (quartic trap case), and number of bosons is 600 . The values of $k_{B} T_{0}$ for these cases are $0.18,1.187$, and 3.19 , respectively.

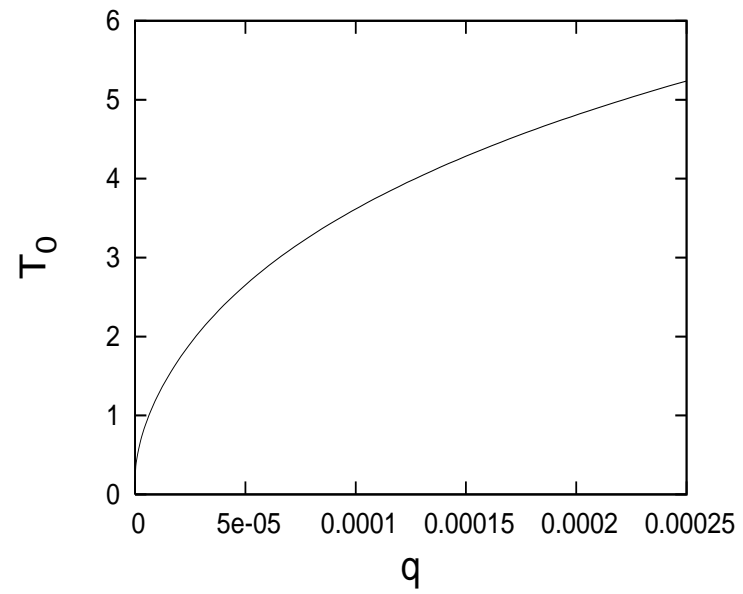

Fig. 9. Quartic potential strength dependence of condensation temperature for 600 bosons in a $2 \mathrm{~d}$ square lattice of size 100 $\times 100$

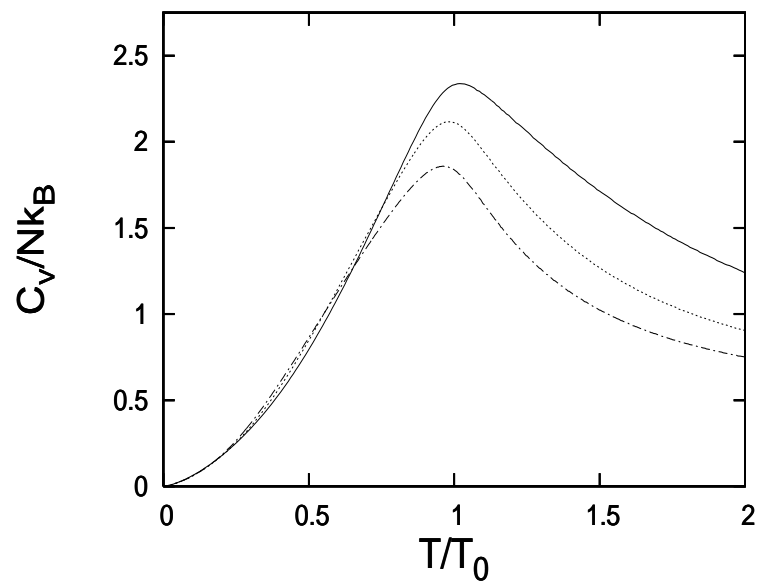

Fig. 10. Temeperature dependence of specific heat of 600 bosons in a $2 \mathrm{~d}$ lattice of size $100 \times 100$ in a quartic potential of stregnths $q=5 \times 10^{-5}$ (solid line), $0.10 \times 10^{-4}$ (dotted), and $0.25 \times 10^{-4}$ (dash-dot). The values of $k_{B} T_{0}$ for these cases are $1.187,1.817$, and 2.421 , respectivley.
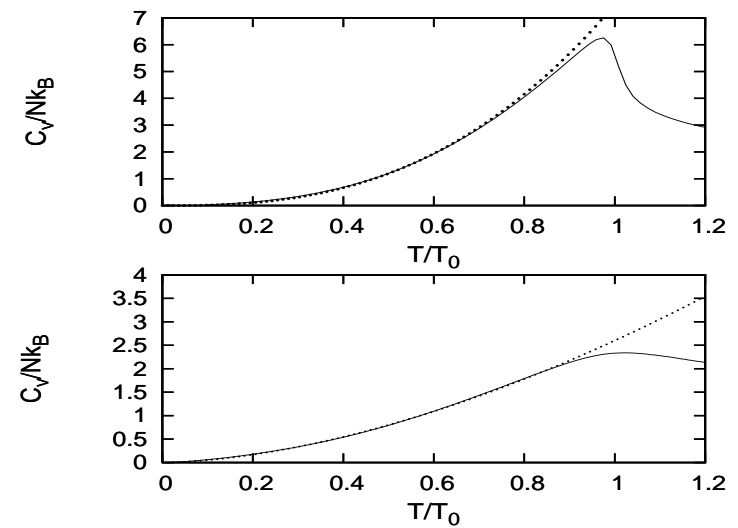

Fig. 11. Fits to low temperature specific heats. 2d (bottom panel): the dotted line is a plot of $2.6 \times\left(T / T_{0}\right)^{1.7} \cdot 3 \mathrm{~d}$ (top panel): the dotted line is a plot of $7.5 \times\left(T / T_{0}\right)^{2.65}$ 


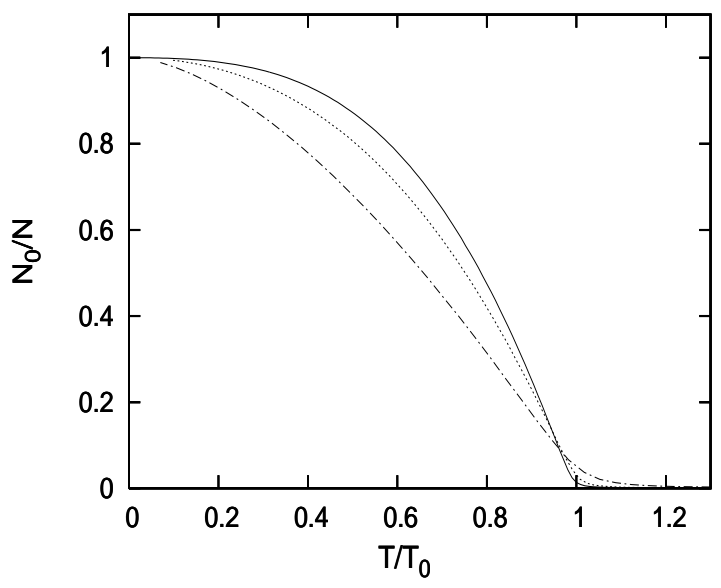

Fig. 12. Comparison of the growth of condensate fraction of lattice bosons in 3d: pure lattice bosons (dash-dot), lattice bosons in a quartic trap of strength $q=10^{-5}$ (dotted), and and lattice bosons in a harmonic trap of strength $k=0.01$ (solid). The lattice size used is $70 \times 70 \times 70$ and number of bosons is 2500 . The values of $k_{B} T_{0}$ for these cases are 0.285 , 1.23 , and 2.08 , respectively.

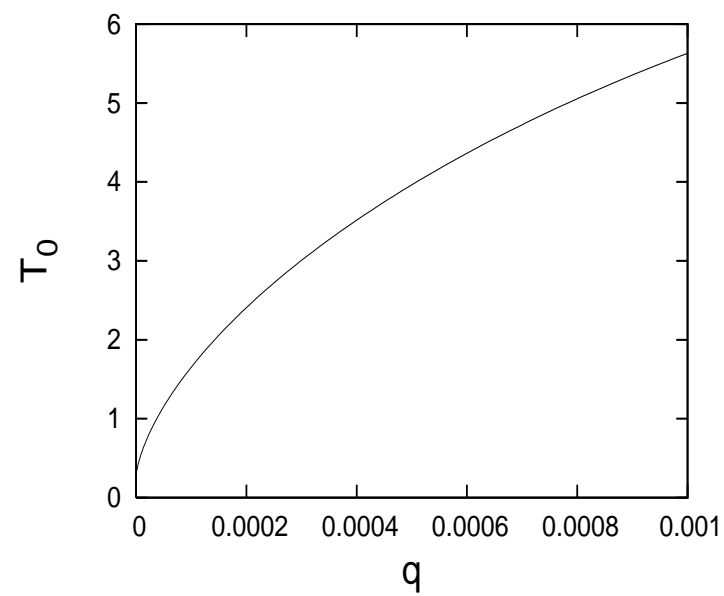

Fig. 13. Quartic potential strength dependence of condensation temperature for 2500 bosons in a $3 \mathrm{~d}$ square lattice of size $70 \times 70 \times 70$.

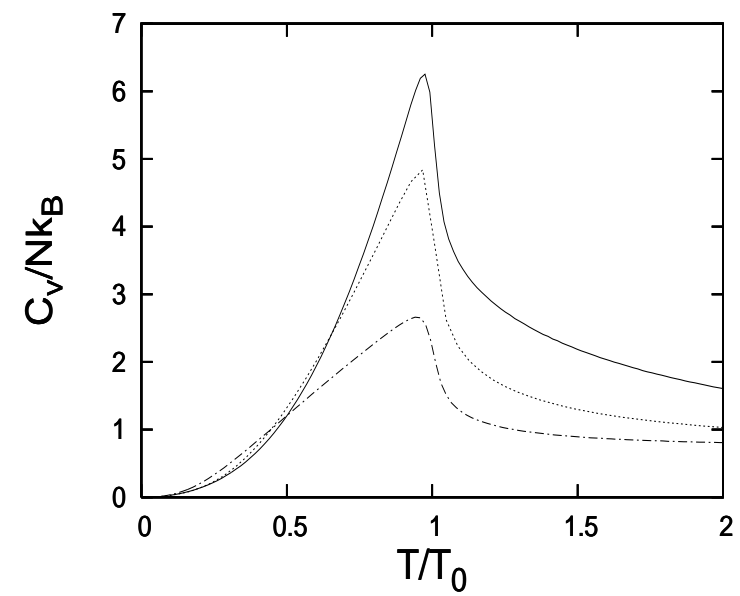

Fig. 14. Temeperature dependence of specific heat of 2500 bosons in a $3 \mathrm{~d}$ lattice of size $70 \times 70 \times 70$ in a quartic potential of stregnths: $q=10^{-5}$ (solid line), $10^{-4}$ (dots), and $10^{-3}$ (dash-dot). The values of $k_{B} T_{0}$ for these cases are 1.23, 2.48, and 5.63, respectivley. 